\title{
Organic field-effect transistors with molecularly doped polymer gate buffer layer
}

\section{$\operatorname{AUTHOR}(\mathrm{S}):$}

Yamagishi, Yuji; Noda, Kei; Yamada, Hirofumi; Matsushige, Kazumi

\section{CITATION:}

Yamagishi, Yuji ... [et al]. Organic field-effect transistors with molecularly doped polymer gate buffer layer. Synthetic Metals 2012, 162(21-22): 1887-1893

\section{ISSUE DATE:}

2012-12

URL:

http://hdl.handle.net/2433/164743

\section{RIGHT:}

(c) 2012 Elsevier B.V.; この論文は出版社版でありません。引用の際には 出版社版をご確認ご利用ください。; This is not the published version. Please cite only the published version. 


\title{
Organic field-effect transistors with molecularly doped polymer gate buffer layer
}

\author{
Yuji Yamagishi*, Kei Noda, Hirofumi Yamada, Kazumi Matsushige \\ Department of Electronic Science and Engineering, Kyoto University, \\ Katsura, Nishikyo, Kyoto 615-8510, Japan \\ *Corresponding author. E-mail address: y.yamagishi@ piezo.kuee.kyoto-u.ac.jp (Y. Yamagishi) \\ and nodakei@kuee.kyoto-u.ac.jp (K. Noda).
}

\begin{abstract}
(Abstract)
Organic field-effect transistors (OFETs) with molecularly doped poly(methylmethacrylate) (PMMA) gate buffer layer were newly investigated. Acceptor doped PMMA buffer layers with a thickness of $8 \mathrm{~nm}$ were deposited onto $\mathrm{SiO}_{2}$ gate insulator by spin-coating methylethylketone solution containing both PMMA and molecular dopants such as tetrafluorotertacyanoquinodimethane (F4TCNQ). Gate threshold voltage shifts in positive direction were commonly observed for both p-channel and n-channel transistors with F4TCNQ doped PMMA gate buffer layer. In p-channel pentacene thin-film transistors, higher dopant ratio led to the increase in the effective hole mobility due to hole doping caused by charge transfer between pentacene and F4TCNQ molecules. In n-channel transistors based on 1,4,5,8-naphthalenetetracarboxylicdianhydride (NTCDA) and copper hexadecafluorophthalocyanine $(\mathrm{F} 16 \mathrm{CuPc}), \mathrm{F} 4 \mathrm{TCNQ}$ molecules doped into the PMMA layer behave as electron traps and NTCDA transistors are more susceptible to acceptor dopants than $\mathrm{F} 16 \mathrm{CuPc}$ ones. This phenomenon in the n-channel transistors can be explained by energetic differences between unoccupied molecular orbital (LUMO) levels of host organic semiconductor and dopant molecules.
\end{abstract}

\section{(Keywords)}

Organic field-effect transistor, Molecularly doped gate buffer layer, Hole doping, Electron trap, Threshold voltage shift

\section{Introduction}


Organic field-effect transistors (OFETs) have been intensely studied for developing printable electronics and functional applications such as sensing devices [1-3]. In order to put OFETs into practical use, control of semiconductor/gate-insulator interface conditions is one of the most important issues because behaviors of carriers injected into semiconductor layer from source electrode are mainly controlled by interface conduction. Therefore fabrication of well-ordered semiconductor/insulator interface has been attempted so far with a variety of passivation techniques. For example, inserting thin layer $(\sim 10 \mathrm{~nm})$ of polymers without hydroxyl groups (-OH), e.g. poly(methylmethacrylate) (PMMA), into semiconductor/insulator interface is quite effective for reducing electron traps on insulator surface and widely used for enhancement of n-channel operation [4-7]. It is well known that silanol $(\mathrm{SiOH})$ groups can work as strong electron attracting species when silicon dioxide $\left(\mathrm{SiO}_{2}\right)$ is employed as gate insulator, and then polymer buffer layer is utilized for suppressing electron traps deriving from SiOH. Another approach toward channel interface control in OFETs is surface modification of gate insulator using chemically-modified self-assembled monolayer (SAMs) $[8,9]$ and molecular dopants $[10,11]$, which modulates carrier accumulation/depletion in the transistor channel and causes a large shift in threshold voltage $\left(V_{\mathrm{TH}}\right)$. However, in the case of chemically-modified SAMs, it is hard to adjust the number density of functional groups in the SAMs because SAM molecules cover gate insulator surfaces uniformly in a wide area and this makes it difficult to control threshold voltage of OFETs precisely. An alternative choice is inserting molecular dopant layers which are usually deposited by thermal evaporation. For instance, a few monolayers of tetrafluorotetracyanoquinodimethane (F4TCNQ) and tetracyanoquinodimethane (TCNQ) are commonly used for control of transistor performance with interface modification [10-12]. But, this method also has some difficulties in controlling the amount of dopant 
evaporation accurately and often results in poor reproducibility of device performances. Under these circumstances, we propose a new approach for control of OFET characteristics by combining the methods of molecular doping and ultrathin polymer buffer layer which we have demonstrated in previous reports [13,14]. In this method, molecularly doped polymer buffer layer was fabricated onto gate insulator surfaces by spin-coating of solution containing a mixture of dielectric polymer and molecular dopant. This simple wet process allows us to set the accurate mixing ratio of inactive polymer to dopant, enabling continuous control of OFET characteristics such as threshold voltage. In order to confirm our idea, we fabricated and characterized OFETs with molecularly doped gate buffer layers composed of typical organic semiconductor and dopant materials in this study. Based on the results, effects of the doping on OFET characteristics were discussed in detail.

\section{Sample preparation}

Chemical structures of PMMA, F4TCNQ and TCNQ are shown in Fig.1 (a), (b) and (c). Top-contact configuration of OFET in Fig.1 (d) was fabricated as follows. PMMA (Mw 120,000 Aldrich) was purified with a reprecipitation technique [13,14]. Both sublimed F4TCNQ and TCNQ were purchased from Tokyo Chemical Industry Co., Ltd. and employed as dopants. PMMA and each of the dopants were dissolved in methylethylketone (2-3 mg ml ${ }^{-1}$ for PMMA and $0.14-3 \mathrm{mg} \mathrm{ml}^{-1}$ for dopant) and prepared as a mixture solution in advance. A molecularly doped PMMA buffer layer was deposited by spin-coating the solution onto a heavily doped silicon wafer which has a thermally grown $\mathrm{SiO}_{2}$ with a nominal thickness of $300 \mathrm{~nm}$, and baked for $10 \mathrm{~min}$ at $393 \mathrm{~K}$ with a hot plate. A F4TCNQ film, which was prepared for comparison, was also fabricated by spin-coating the 
methylethylketone solution containing F4TCNQ onto $\mathrm{SiO}_{2}$. Ellipsometric measurements were performed for PMMA films using a DHA-FX (Mizojiri Optical Ltd.).

We chose pentacene, 1,4,5,8-naphthalenetetracarboxylicdianhydride (NTCDA), and copper hexadecafluorophthalocyanine (F16CuPc) as organic semiconductor materials. Pentacene (Aldrich) and F16CuPc (Aldrich) were purified by train sublimation before deposition. Sublimed NTCDA was purchased from Tokyo Chemical Industry Co., Ltd. Organic semiconducting layers were deposited by vaccum evaporation onto doped PMMA buffer layers with various dopant concentrations in a vacuum of $1.0 \times 10^{-4} \mathrm{~Pa}$. The thickness of the organic semiconductor films and the substrate temperature were set to be $50 \mathrm{~nm}$ and room temperature, respectively. After that, $25 \mathrm{~nm}$ of gold was deposited onto the organic semiconductor layer through a shadow mask to form source-drain electrodes. The channel length and width were $50 \mu \mathrm{m}$ and $1 \mathrm{~mm}$, respectively. Electrical measurements of OFETs were conducted in a vacuum $\left(1.0 \times 10^{-1} \mathrm{~Pa}\right)$ with a KEITHLEY 4200-SCS semiconductor parameter analyzer. Effective mobility $(\mu)$ and threshold voltage $\left(\mathrm{V}_{\mathrm{TH}}\right)$ were obtained from transfer characteristics (drain-source current $\left(\mathrm{I}_{\mathrm{D}}\right)$ vs. gate voltage $\left(\mathrm{V}_{\mathrm{G}}\right)$ ) in the saturation regime. Observation of surface morphologies of the PMMA layers and organic semiconductor layers was performed with an atomic force microscopy (AFM) (JSPM-5200, JEOL).

\section{Results and discussion}

\subsection{Surface morphology of molecularly doped PMMA layers and organic semiconductor films}

Figure 2 shows surface structures of molecularly doped PMMA layers observed with AFM. All specimens reveal 
considerably flat surfaces and the root-mean-square (rms) roughness values of undoped, F4TCNQ-doped and TCNQ-doped are $0.24 \mathrm{~nm}, 0.29 \mathrm{~nm}$ and $0.39 \mathrm{~nm}$ respectively. A previous study has shown that the gate dielectric surface rms roughness of more than $0.5 \mathrm{~nm}$ considerably reduced the carrier mobility in pentacene FETs due to the reduction of pentacene grain size and crystallinity [15]. Since the surface rms roughness values of all the PMMA layers in Fig. 2 were smaller than $0.5 \mathrm{~nm}$, molecularly doped PMMA layers in this study probably do not affect the film growth of semiconductor materials on the gate dielectric surfaces.

Surface morphologies of organic semiconductor films deposited on undoped and doped PMMA were also examined with AFM. In the case of NTCDA (Fig.3), numerous granular grains were formed on both undoped and F4TCNQ-doped PMMA. Although both of the NTCDA films have somewhat rough surface morphology, obvious structural difference between the NTCDA films was not observed. F16CuPc films have smoother surfaces compared with those of the NTCDA films as shown in Fig.4. Pentacene films on doped and undoped PMMA showed similar dendritic grain growth which is typical of pentacene as shown in Fig.5 (a), (b) and (c). Here, it is noteworthy that molecular doping in PMMA gate buffer layer does not cause any structural difference in these semiconductor layers. On the other hand, pentacene films deposited on bare $\mathrm{SiO}_{2}\left(\mathrm{Fig} .5\right.$ (d)) and $\mathrm{SiO}_{2}$ coated with F4TCNQ (Fig.5 (e)) show much smaller grains in comparison with the pentacene films on the PMMA layers. Since it is known that larger grains often give rise to higher carrier mobility in OFETs [16], surface structures of the organic semiconductor films should be identical regardless of molecular doping for discussing the charge transfer interaction between semiconductor layer and dopant in detail. Besides, in terms of controlling the threshold voltage in OFETs, unexpected change in field-effect mobility by molecular doping should be avoided. From these reasons, 
the molecularly doped PMMA buffer layer is thought to be useful for altering the interface between semiconductor

and insulator in a controllable manner.

\subsection{OFETs with molecularly doped PMMA buffer layers}

Transfer characteristics of pentacene and F16CuPc OFETs with undoped and doped PMMA buffer layers are presented in Fig.6. The thickness of the PMMA buffer layers was almost the same ( $8 \pm 1.5 \mathrm{~nm})$. The dopant ratio of F4TCNQ (TCNQ) was 20.0 (25.6) wt\%. Here, number densities of each dopant in PMMA buffer layer were set to be the same on the assumption that the density of PMMA and each of the dopants are $1.2 \mathrm{~g} / \mathrm{cm}^{3}$ and $1.4 \mathrm{~g} / \mathrm{cm}^{3}$, respectively. In both Fig.6 (a) and (b), OFETs with F4TCNQ-doped buffer layers show clear doping effects, especially in $V_{\mathrm{TH}}$ shift, while on the other hand TCNQ doped devices show smaller characteristic changes compared with F4TCNQ doped ones. $V_{\mathrm{TH}}$ in pentacene OFET becomes shifted in the positive direction with F4TCNQ-doped buffer layer and this is possibly due to hole doping caused by electron transfer between HOMO (Highest Occupied Molecular Orbital) of pentacene (5.0 eV) [20] to LUMO (Least Unoccupied Molecular Orbital) of F4TCNQ (5.2 eV) [17] as depicted in Fig.6 (c), resulting in $V_{\mathrm{TH}}$ shift in positive direction. On the other hand, pentacene OFETs with TCNQ-doped buffer layers show no clear characteristic change. This is presumably because electron affinity of TCNQ $(4.2 \mathrm{eV})$ is smaller than that of F4TCNQ, and charge transfer between pentacene and TCNQ molecules does not occur sufficiently.

In F16CuPc OFETs, $V_{\mathrm{TH}}$ is largely increased with F4TCNQ-doped buffer layer, but TCNQ-doped OFETs revealed smaller changes in device performance. In n-channel transport of F16CuPc transistors, F4TCNQ 
molecules with an electron affinity $(5.2 \mathrm{eV})$ larger than that of $\mathrm{F} 16 \mathrm{CuPc}(4.7 \mathrm{eV})$ [19] work as electron traps and electrons existing at LUMO level of $\mathrm{F} 16 \mathrm{CuPc}$ under device operation are prone to move to LUMO level of F4TCNQ. However, TCNQ molecules cannot work as electron traps for F16CuPc layer due to smaller electron affinity of TCNQ $(4.2 \mathrm{eV})$. We would like to note that no electron exist in the LUMO level of the semiconductor except under device operation. Therefore, mechanisms in which accepter dopants cause $V_{\mathrm{TH}}$ shifts for p-channel and n-channel OFETs should be clearly distinguished.

\subsection{Dependence of OFET characteristics on F4TCNQ concentration in PMMA buffer layers}

OFET devices with PMMA gate buffer layers doped at various F4TCNQ concentrations were fabricated. The thickness of the PMMA buffer layers in all devices was also about $8 \mathrm{~nm}$. First, transfer characteristics of pentacene OFETs with the PMMA layers doped at different ratios of F4TCNQ are shown in Fig.7 (a) and parameters obtained from these data are listed in Table 1. In pentacene OFETs, $V_{\mathrm{TH}}$ was shifted in positive direction with F4TCNQ doping, and the amount of the positive shift correlates with the dopant ratio. Moreover, the values of the hole mobility in pentacene OFETs were also increased with the larger amount of dopant in the buffer layer, and it approximately doubled for the dopant ratio of $20.0 \mathrm{wt} \%\left(0.19 \mathrm{~cm}^{2} / \mathrm{Vs}\right)$ compared to the undoped one $\left(0.10 \mathrm{~cm}^{2} / \mathrm{Vs}\right)$ as shown in Table 1. This dependence of the hole mobility on the dopant concentration can be explained as follows. Charge transport in organic semiconductor thin films is governed by hopping between localized states in the tail of an energy distribution [16,21]. At higher dopant concentrations, deeper localized states are filled with increased number of charge carriers and the charge transport takes place at higher energy levels. Therefore the energy center 
of the localized states and hopping rate for holes become higher as the dopant concentration is increased, so that effective hole mobility is improved. Actually, when the dopant ratio was changed from 0.0 to $11.1 \mathrm{wt} \%, V_{\mathrm{TH}}$ was shifted in positive direction from -23.7 to $-18.7 \mathrm{~V}$ in contrast to the relatively small increase in mobility from 0.10 to $0.11 \mathrm{~cm}^{2} / \mathrm{Vs}$. Here, donated holes are mainly employed to fill deeper localized states which are associated with transistor channel formation, and this contributes to the $V_{\mathrm{TH}}$ shift. Successively, when the dopant ratio was increased to $20.0 \mathrm{wt} \%$, the hole mobility was increased up to $0.19 \mathrm{~cm}^{2} / \mathrm{Vs}$, with a small $V_{\mathrm{TH}}$ shift of $1.1 \mathrm{~V}$. This means that further doped holes are used to fill shallower states and improve the effective hole mobility. Finally, higher dopant ratio up to $33.3 \mathrm{wt} \%$ led to drastic increase of $I_{\mathrm{D}}$ and complete normally-on operation possibly due to the excess hole doping directly to pentacene HOMO band.

Next, n-channel transport properties in F16CuPc and NTCDA OFETs with PMMA layers at different ratios of F4TCNQ were also examined. $I_{\mathrm{D}}-V_{\mathrm{G}}$ curves of these n-channel OFETs are represented in Fig.7 (b) and (c), and parameters obtained from these data are summarized in Table 2 and 3. Here $N$ is an estimated upper limit of charge trap density at semiconductor/gate-insulator interface and calculated using the following equation [22,23].

$$
N=\left[\frac{q S \log (e)}{k T}-1\right] \frac{C_{\mathrm{OX}}}{q}
$$

where $q$ the elementary charge, $S$ the subthreshold swing, $k$ the Boltzmann constant, $T$ the temperature, and $C_{\mathrm{OX}}$ the gate capacitance per unit area.

In the case of F16CuPc OFETs, the larger doping ratio of F4TCNQ gave rise to the increase in the $V_{\mathrm{TH}}$ and the subthreshold swing as shown in Fig. 8 and Table 2 . The $V_{\mathrm{TH}}$ shift was relatively small at lower dopant ratios of 20.0 and $33.3 \mathrm{wt} \%$, however $V_{\mathrm{TH}}$ increased up to $19.7 \mathrm{~V}$ at the dopant ratio of $50.0 \mathrm{wt} \%$. The subthreshold swing, 
which is usually related to localized states in the bulk layer or at the semiconductor/dielectric interface [24,25], also increased with increasing the amount of dopant. It seems that a certain percentage of F4TCNQ molecules embedded in the buffer layers might be exposed on the surface particularly at higher doping ratios, and they can work as the localized states at the semiconductor/dielectric interface. The degradation of electron mobility with increasing amount of dopant (Table 2) is also explainable by the change in energy distribution of localized transport states. F4TCNQ molecules doped into the buffer layers give rise to deep electron trap states. Actually, deep electron trap density $(N)$ is up to $10^{13} \mathrm{~cm}^{-2}$ with F4TCNQ doping. In such cases, the charge transition rate between localized states becomes smaller, resulting in lower electron mobility.

In the case of NTCDA OFETs, smaller amounts of F4TCNQ doped into PMMA (20.0, 11.1 and 5.9 wt\%) caused obvious positive shift of $V_{\mathrm{TH}}$ and increase of subthreshold swing, and additional F4TCNQ doping into PMMA (more than $20.0 \mathrm{wt} \%$ ) disabled transistor operation of NTCDA OFETs. On the other hand, F16CuPc OFETs which have PMMA buffer layers doped with the ratio of 50.0 or $33.3 \mathrm{wt} \%$ still maintained n-channel operation as shown in Fig. 7 (b). These differences in n-channel transport can be attributed to the energetic difference between LUMO levels of NTCDA and F16CuPc as shown in Fig.9. The energetic difference between LUMO of NTCDA (4.0 eV) and F4TCNQ $(5.2 \mathrm{eV})$ is larger than that of F16CuPc $(4.7 \mathrm{eV})$ and F4TCNQ $(5.2 \mathrm{eV})$. Therefore, the function of F4TCNQ as carrier electron traps for NTCDA should be stronger than that for F16CuPc, confirmed by more outstanding characteristic changes in NTCDA OFETs with F4TCNQ doping to PMMA layer.

\subsection{Device stability measurements of OFETs with F4TCNQ doped PMMA buffer layers}


Device stability of OFETs with undoped and F4TCNQ doped buffer layers was examined by repeating forward and backward gate voltage sweeps. In this experiment, dopant ratio was set to be $20 \mathrm{wt} \%$ for all the samples. Fig. 10 shows transfer characteristics of the OFETs at the initial state and after 20 cycles of gate voltage sweep. As shown in Fig. 10(a), $I_{\mathrm{G}}-V_{\mathrm{D}}$ curves of the pentaceme OFET with F4TCNQ doped PMMA layer revealed no shift even after the repetitive gate voltage sweeps, while a small negative shift in $I_{\mathrm{G}}-V_{\mathrm{D}}$ curve owing to the sweeps was observed for the device with undoped PMMA layer. Operational stability of pentacene OFETs against bias stress can be improved by utilizing acceptor-doped gate buffer layers possibly because of the trap-filling effects with hole doping in pentacene layers.

As for n-channel transistors, transfer characteristics of the F16CuPc OFET with F4TCNQ doped PMMA layer were not degraded with the repetitive gate voltage sweeps, as shown in Fig. $10(\mathrm{~b})$. In contrast, $I_{\mathrm{G}}-V_{\mathrm{D}}$ curve of the NTCDA transistor with F4TCNQ doped PMMA layer was shifted in positive $V_{\mathrm{D}}$ direction after 20 cycles of gate bias sweep, as shown in Fig. 10(c). In particular, this shift was prominent in the subthreshold region. These results on the device stability of n-channel OTFTs suggest that excessive doping can induce instability of n-channel operation with bias stress. Moreover, as shown in Fig. 7(a), excessive doping in p-channel devices also caused the drastic increase of off current and degraded the function as field-effect devices. Therefore, proper dopant ratios should be considered for preventing instability of device operation according to the choice of host organic semiconductor and dopant materials. 


\section{Conclusions}

OFETs with molecularly doped PMMA gate buffer layers were investigated in this work. Ultrathin polymer gate buffer layers doped with various dopants were prepared by simple spin-coating method. Acceptors doped into the buffer layers caused characteristic changes especially in $V_{\mathrm{TH}}$ for both p-channel and n-channel OFETs. The observed phenomena in transistor characteristics are considered in terms of hole doping and electron trapping behaviors caused by acceptor dopants. Utilization of molecularly doped gate buffer layers proposed in this work enables us not only to control OFET characteristics, but also to investigate carrier behaviors at the semiconductor/insulator interface.

\section{Acknowledgements}

The authors thank the supports from Grant-in-aid for scientific research (KAKENHI, No. 24656205) from the Japan Society for the Promotion of Science (JSPS). This work was also supported by Joint Research and Development Project of International Standards funded by the Ministry of Economy, Trade and Industry of Japan (METI).

\section{References}

[1] T. Sekitani, U. Zschieschang, H. Klauk, T. Someya, Nat. Mater. 9 (2010) 1015-1022. 
[2] Y. Guo, G. Yu, Y. Liu, Adv. Mater. 22 (2010) 4427-4447.

[3] P. Lin, F. Yan, Adv. Mater. 24 (2011) 34-51.

[4] L.L. Chua, J. Zaumseil, J.F. Chang, E.C.W. Ou, P.K.H. Ho, H. Sirringhaus, R.H. Friend, Nature 434 (2005) 194-199.

[5] J. -W. Chang, W. -L. Hsu, C. -Y. Wu, T. -F. Guo, T. -C. Weh, Org. Electron. 11 (2010) 1613-1619.

[6] S. Lee, B. Koo, J. Shin, E. Lee, H. Park, H. Kim, Appl. Phys. Lett. 88 (2006) 162109.

[7] F. -C. Chen, C. -H. Liao, Appl. Phys. Lett. 93 (2008) 103310.

[8] S. Kobayashi, T. Nishikawa, T. Takenobu, S. Mori, T. Shimoda, T. Mitani, H. Shimotani, N. Yoshimoto, S.

Ogawa, Y. Iwasa, Nat. Mater. 3 (2004) 317-322.

[9] M. Halik, H. Klauk, U. Zschieschang, G. Schmid, C. Dehm, M. Schutz, S. Maisch, F. Effenberger, M. Brunnbauer, F. Stellacci, Nature 431 (2004) 963-966.

[10] Y. Abe, T. Hasegawa, Y. Takahashi, T. Yamada, Y. Tokura, Appl. Phys. Lett. 87 (2005) 153506.

[11] J. Li, X. W. Zhang, L. Zhang, K. U. Haq, X. Y. Jiang, W. Q. Zhu, Z. L. Zhang, Solid State Commun. 149 (2009) 1826-1830.

[12] K. Tsukagoshi, K. Shigeto, I. Yagi, Y. Aoyagi, Appl. Phys. Lett. 89 (2006) 113507.

[13] S. Tanida, K. Noda, H. Kawabata, K. Matsushige, Polym. Adv. Technol. 21 (2010) 528-532.

[14] S. Tanida, K. Noda, H. Kawabata, K. Matsushige, Synth. Met. 160 (2010) 1574-1578.

[15] S. E. Fritz, T. W. Kelley, C. D. Frisbie, J. Phys. Chem. B 109 (2005) 10574-10577

[16] H. Kleemann, C. Schuenemann, A. A. Zakhidov, M. Riede, B. Lussem, K. Leo, Org. Electron. 13 (2012) 
$58-65$

[17] W. Gao, A. Kahn, Org. Electron. 3 (2002) 53-63.

[18] C. K. Chan, E.-G. Kim, J. -L. Bredas, A. Kahn, Adv. Funct. Mater. 16 (2006) 831-837.

[19] J. H. Park, S. W. Cho, S. H. Park, J. G. Jeong, H. J. Kim, Y. Yi, M. -H. Cho, Synth. Met. 160 (2010) 108-112.

[20] A. Kahn, N. Koch, W. Gao, Polym. Phys. 41 (2003) 2529-2548.

[21] S. Yin, Y. Yang, Y. Lv, Synth. Met. 160 (2010) 1241-1246.

[22] A. Rolland, J. Richard, J. P. Kleider, D. Mencaraglia, J. Electrochem. Soc. 140 (1993) 3679-3683.

[23] M. McDowell, I. G. Hill, J. E. McDermott, S. L. Bernasek, J. Schwartz, Appl. Phys. Lett. 88 (2006) 073505.

[24] N. Kawasaki, Y. Ohta, Y. Kubozono, A. Konishi, A. Fujiwara, Appl. Phys. Lett. 92 (2008) 163307.

[25] B. M. Dhar, R. Ozgun, T. Dawidszyk, A. Andreou, H. E. Katz, Mat. Sci. Eng. R 72(2011) 49.

\section{(Figure captions)}

Fig.1. Chemical structure of (a) poly(methylmethacrylate)(PMMA) (b) tetrafluorotetracyanoquinodimethane (F4TCNQ) and (c) tetracyanoquinodimethane (TCNQ). (d) A device structure of OFET with PMMA buffer layer.

Fig.2 AFM images of (a) undoped, (b) F4TCNQ-doped and (c) TCNQ-doped PMMA. These films were formed by spin-coating. The dopant ratio was fixed at about $50.0 \mathrm{wt} . \%$.

Fig.3 AFM images of NTCDA films on (a) undoped (b) F4TCNQ-doped PMMA. 
Fig.4 AFM images of F16CuPc films on (a) undoped (b) F4TCNQ-doped and (c) TCNQ-doped PMMA.

Fig.5 AFM images of pentacene films on (a) undoped PMMA (b) F4TCNQ-doped PMMA, and (c) TCNQ-doped PMMA. Pentacene films deposited on (d) F4TCNQ films and (e) bare $\mathrm{SiO}_{2}$ are also shown.

Fig.6 Transfer characteristics of (a) pentacene and (b) F16CuPc OFETs with undoped PMMA (green),

TCNQ-doped (blue) and F4TCNQ-doped PMMA (red) buffer layers. The dopant ratio was fixed at about 20.0 wt.\%

(c) Simplified energy level alignment of the materials employed in section 3.3. Numbers noted under the white and

black bars are the LUMO and HOMO levels [17-20]. Arrows shown in the figures are the expected direction of the electron transfer.

Fig.7 Transfer characteristics of OFETs with PMMA buffer layers doped at different F4TCNQ concentrations.

Employed materials are (a) pentacene, (b) F16CuPc and (c) NTCDA. Numbers noted in the graphs are the mass ratios of PMMA and F4TCNQ (PMMA : F4TCNQ).

Fig.8 Dependence of $V_{\mathrm{TH}}$ on the dopant ratio in the F4TCNQ doped PMMA buffer layers. Employed semiconductor materials are pentacene, F16CuPc and NTCDA. 
Fig.9 Simplified energy level alignment of NTCDA, F16CuPc and F4TCNQ [17-20].

Fig,10 Transfer characteristics of OFETs with undoped and F4TCNQ doped gate buffer layers at the initial state and after 20 cycles of gate-sweep measurement. Employed semiconductor materials are (a) pentacene, (b) F16CuPc and (c) NTCDA. 
Fig.1

(a)

$\left(\begin{array}{c}\mathrm{CH}_{2}-\mathrm{CH} \\ \mathrm{CH}_{2} \\ \mathrm{CO} \\ \mathrm{OCH}_{3}\end{array}\right)_{n}$ (b)<smiles>N#CC(C#N)=c1c(F)c(F)c(=C(C#N)C#N)c(F)c1F</smiles>

(c)<smiles>N#CC(C#N)=c1ccc(=C(C#N)C#N)cc1</smiles>

(d)

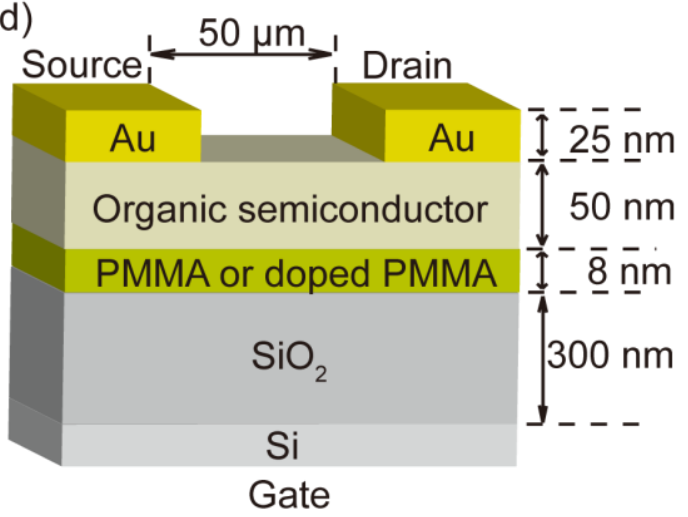


(a)

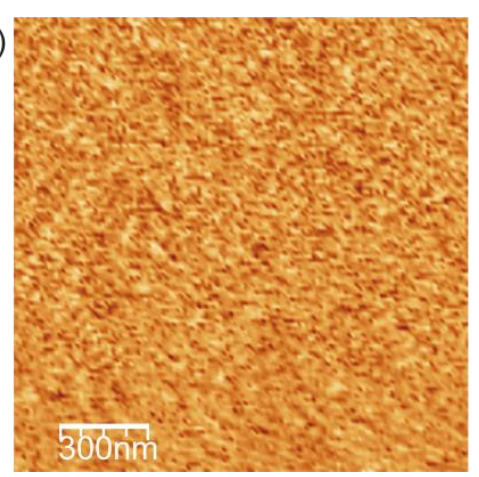

(b)

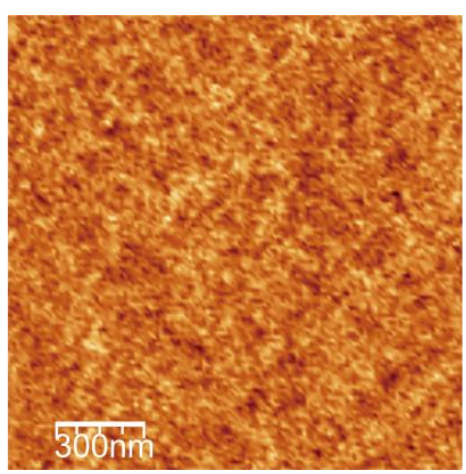

Fig.2

(c)

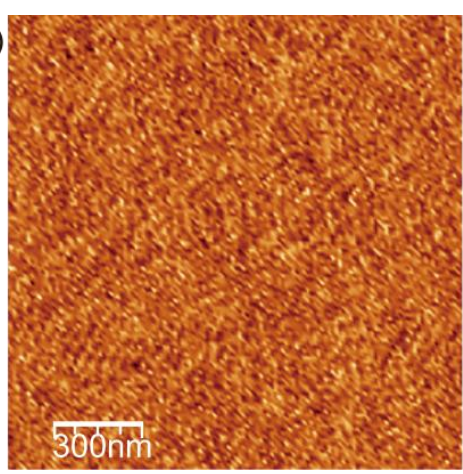


Fig.3

(a)

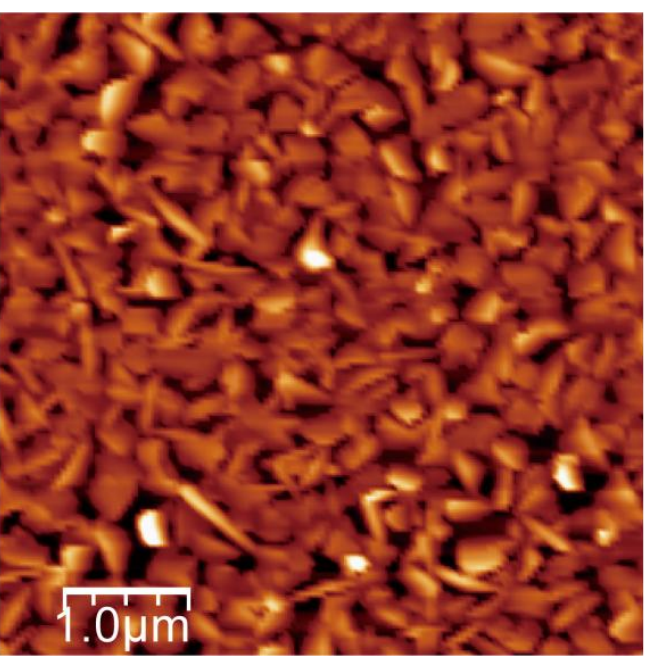

(b)

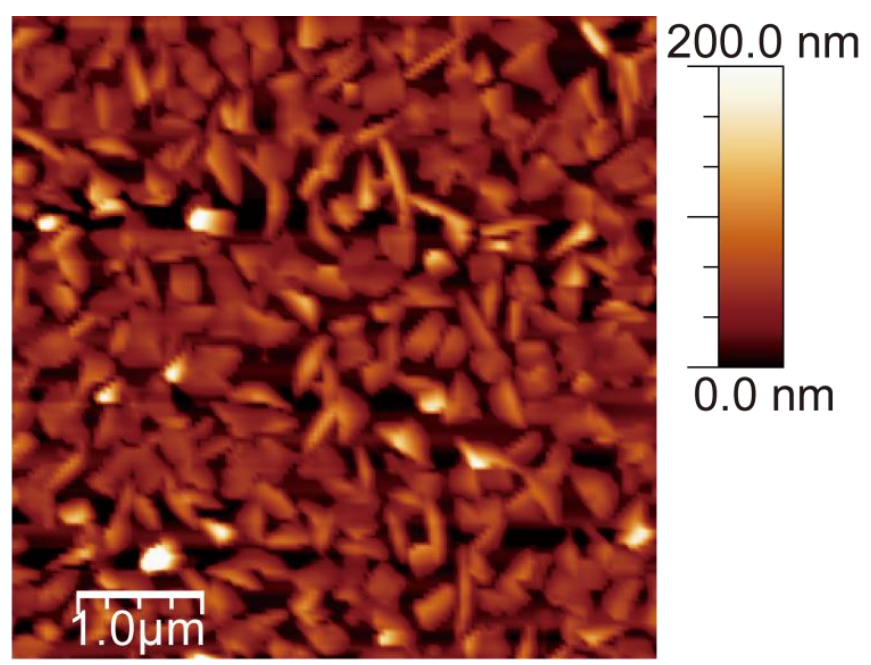


Fig.4

(a)

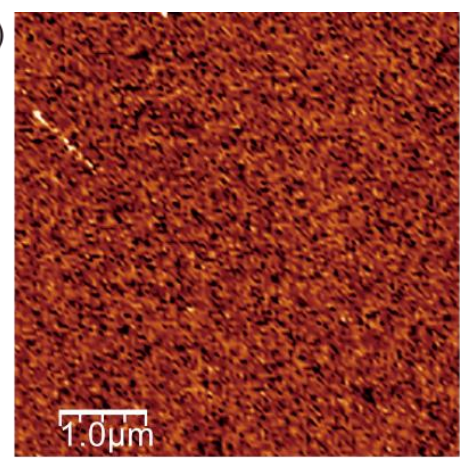

(b)

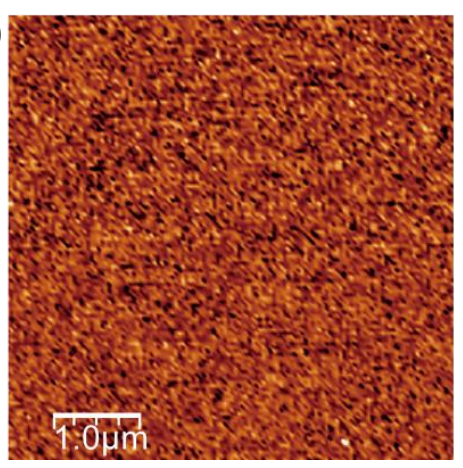

(c)

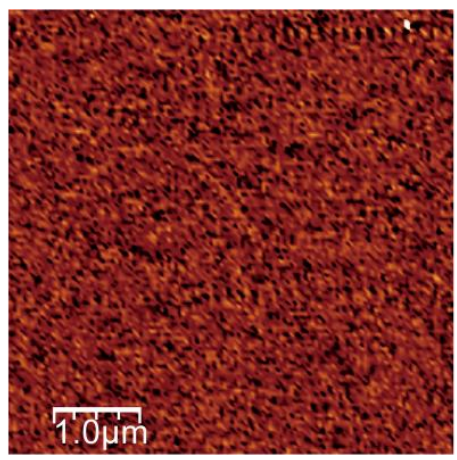

$10.0 \mathrm{~nm}$

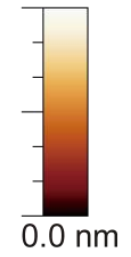


(a)

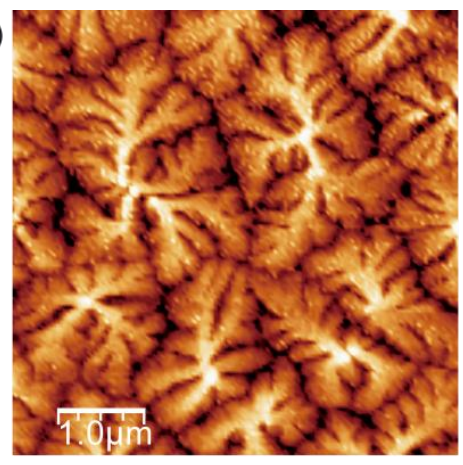

(b)

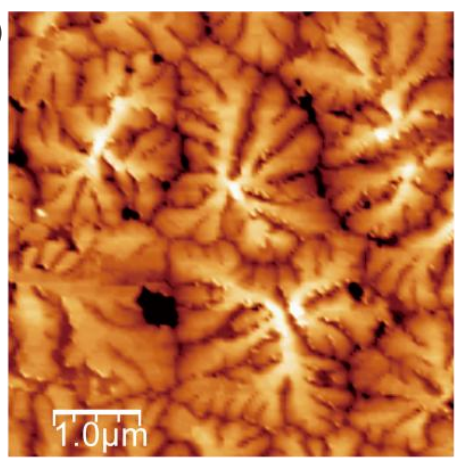

(c)

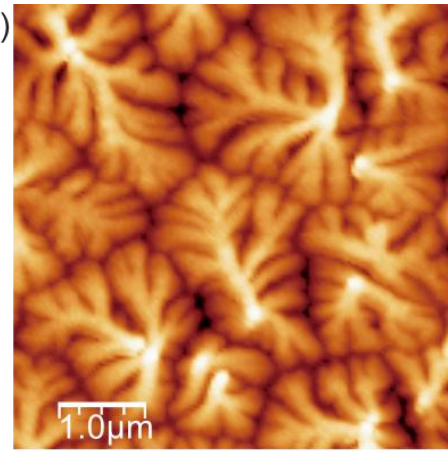

Fig.5

(d)

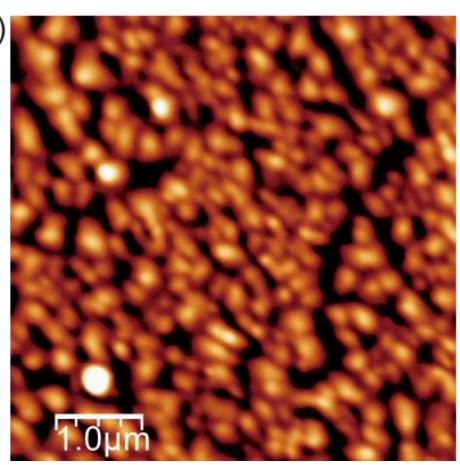

(e)

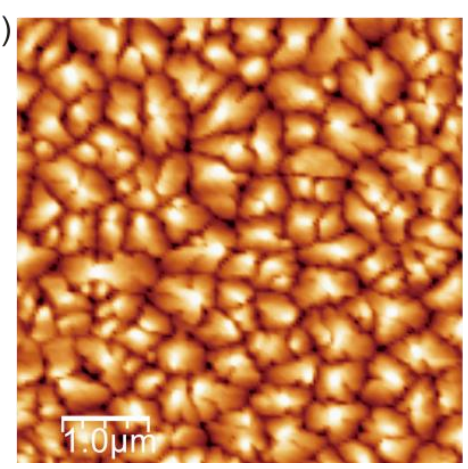

$30.0 \mathrm{~nm}$

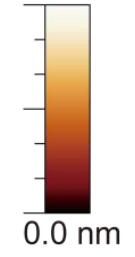




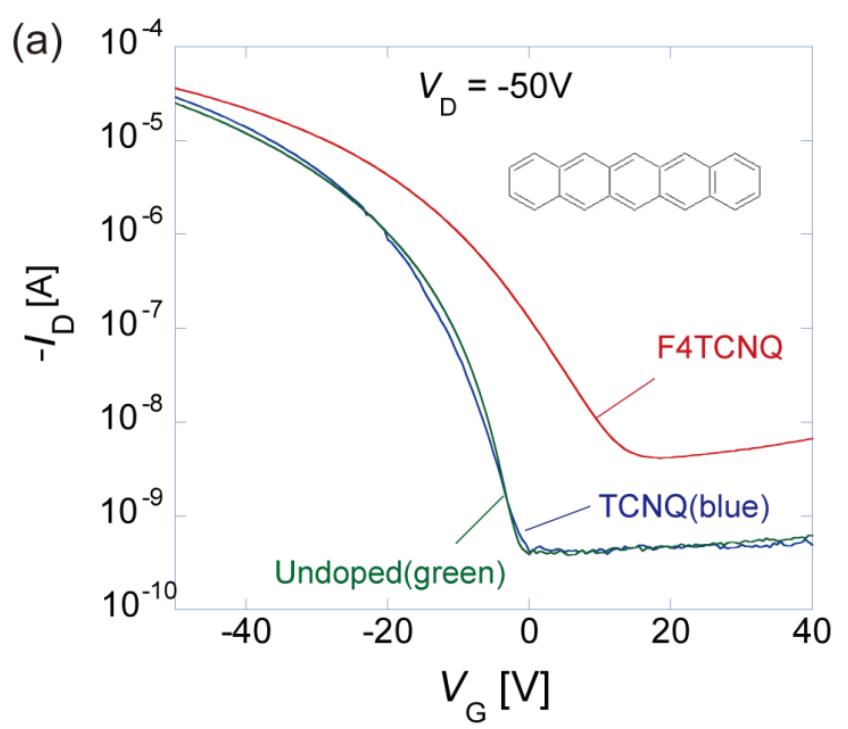

(c)

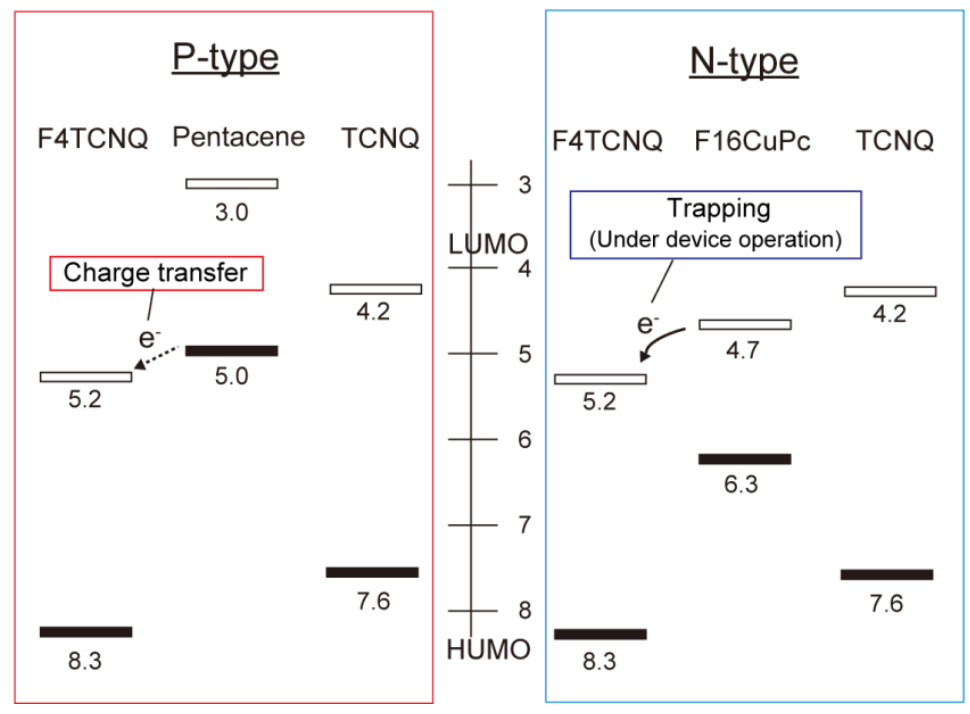

(b) $10^{-6}$

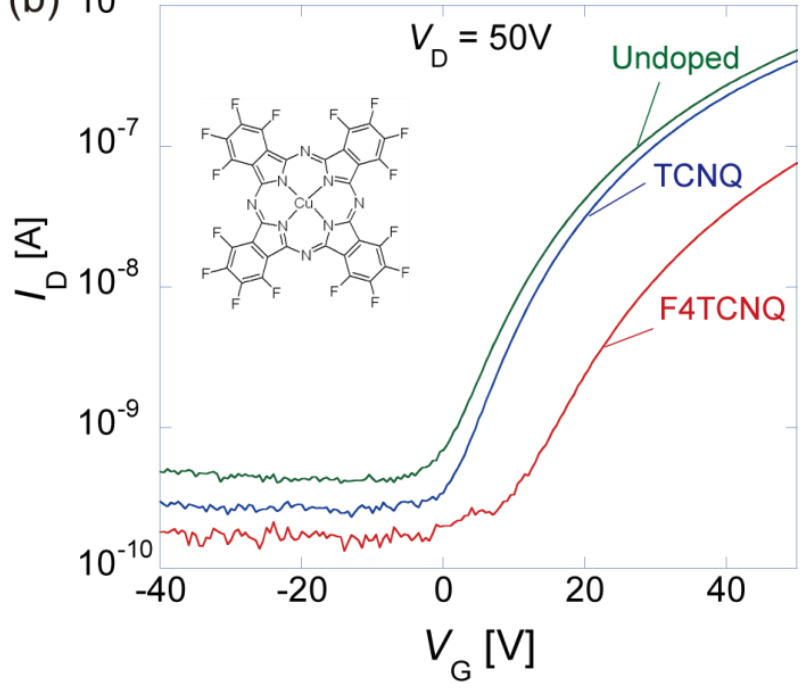

(d)
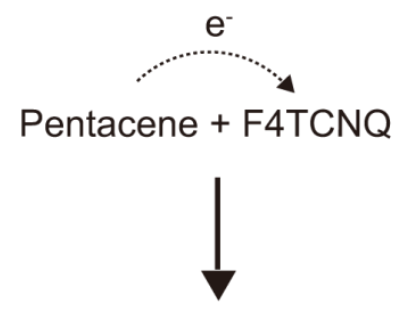

Pentacene ${ }^{\delta^{+}} \ldots$ F $4 \mathrm{TCNQ}^{\delta^{-}}$

Fig.6 
(a) $10^{-2}$

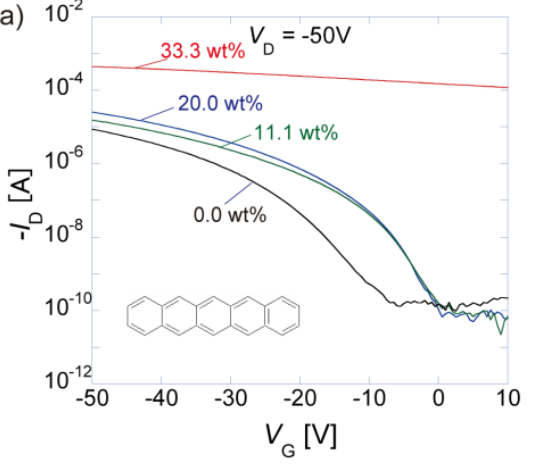

(b)

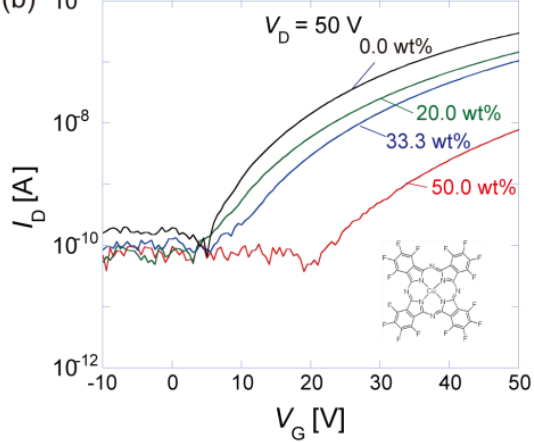

Fig.7

(c) $10^{-6}$

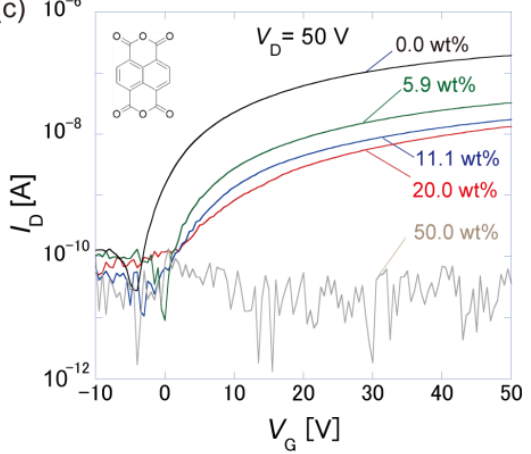




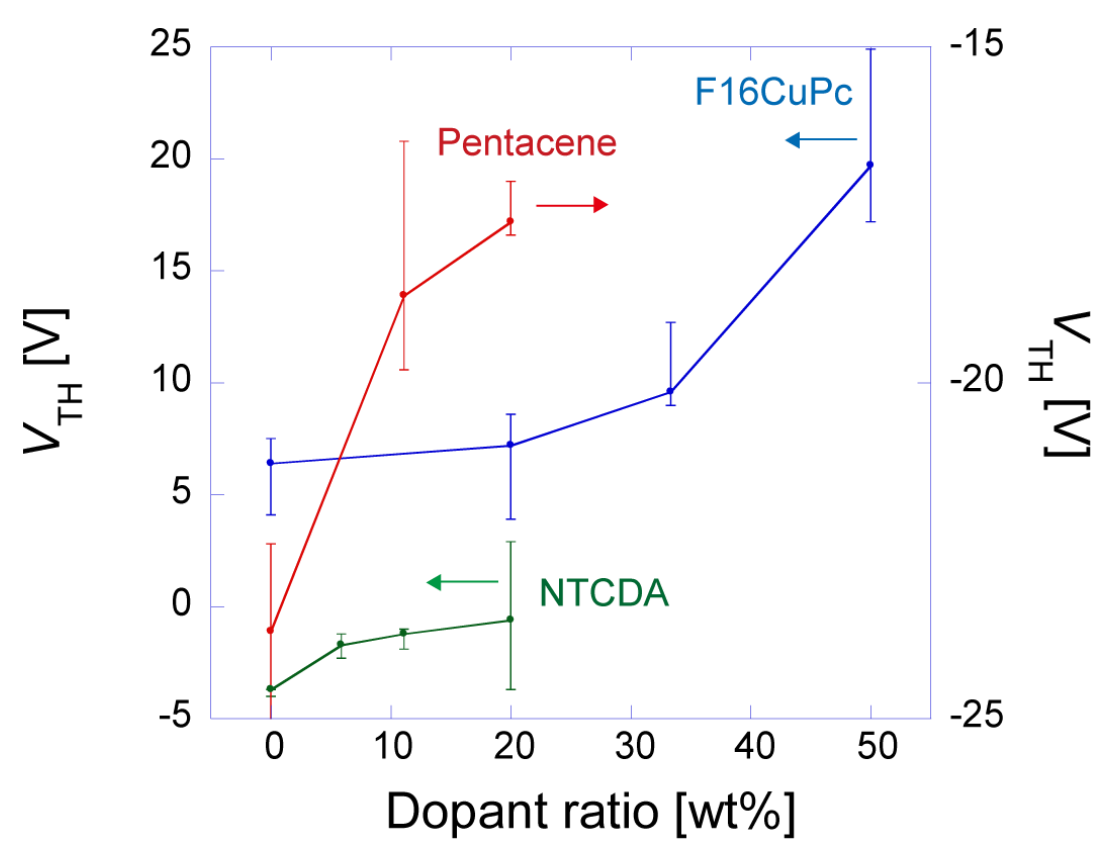

Fig.8 
Fig.9

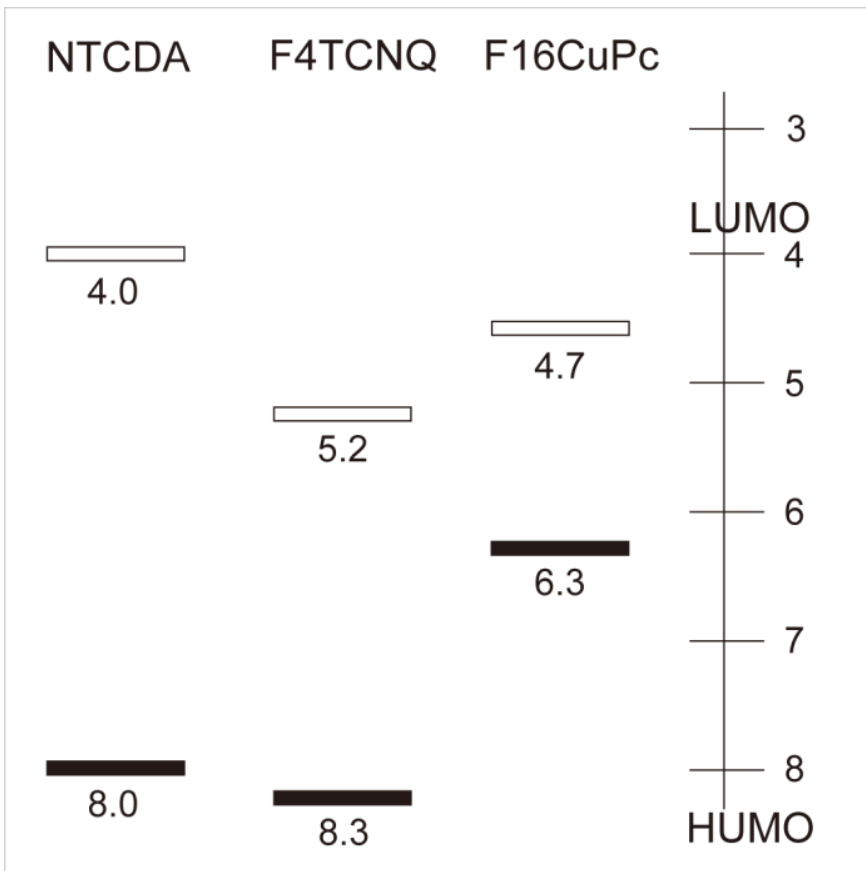


Fig.10
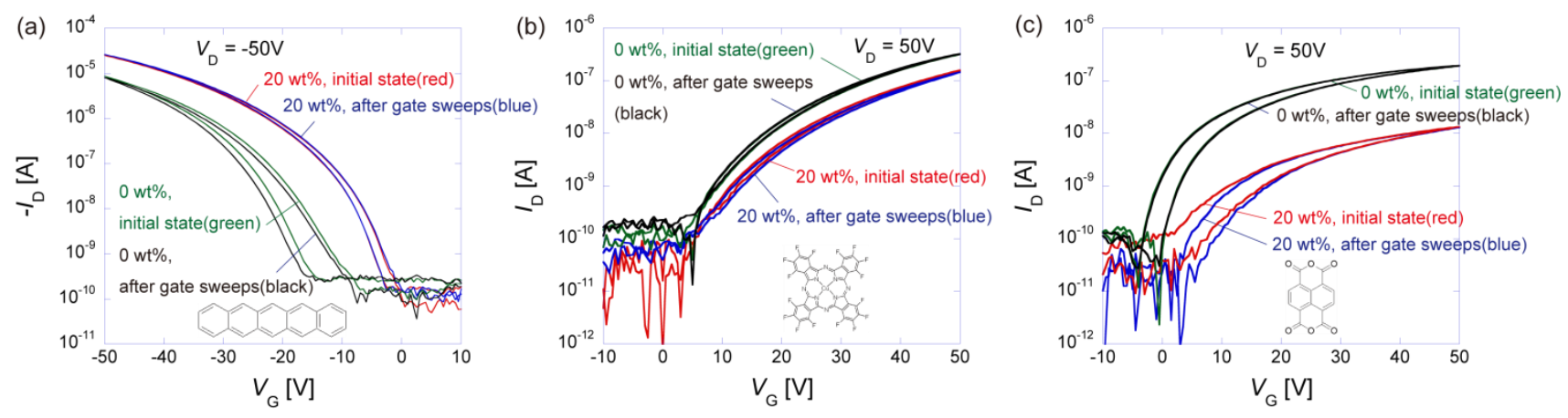


\begin{tabular}{ccc}
\hline \hline Dopant ratio [wt $\%]$ & $\mu\left[\mathrm{cm}^{2} / \mathrm{Vs}\right]$ & $V_{\mathrm{TH}}[\mathrm{V}]$ \\
\hline 20.0 & 0.19 & -17.6 \\
11.1 & 0.11 & -18.7 \\
0.0 & 0.10 & -23.7 \\
\hline \hline
\end{tabular}

Table 1. Parameters obtained from pentacene OFETs with PMMA buffer layers doped at different F4TCNQ concentrations. 


\begin{tabular}{ccccc}
\hline \hline Dopant ratio $[\mathrm{wt} \%]$ & $\mu\left[\mathrm{cm}^{2} / \mathrm{Vs}\right]$ & $V_{\mathrm{TH}}[\mathrm{V}]$ & $S[\mathrm{~V} / \mathrm{dec}]$ & $N\left[/ \mathrm{cm}^{2}\right]$ \\
\hline 50.0 & $4.1 \times 10^{-5}$ & 19.7 & 10.7 & $1.4 \times 10^{13}$ \\
33.3 & $2.5 \times 10^{-4}$ & 9.6 & 9.2 & $1.2 \times 10^{13}$ \\
20.0 & $2.9 \times 10^{-4}$ & 7.2 & 8.2 & $1.0 \times 10^{13}$ \\
0.0 & $6.1 \times 10^{-4}$ & 6.4 & 5.9 & $7.4 \times 10^{12}$ \\
\hline \hline
\end{tabular}

Table 2. Parameters obtained from F16CuPc OFETs with PMMA buffer layers doped at different F4TCNQ concentrations. 


\begin{tabular}{ccccc}
\hline \hline Dopant ratio $[\mathrm{wt} \%]$ & $\mu\left[\mathrm{cm}^{2} / \mathrm{Vs}\right]$ & $V_{\mathrm{TH}}[\mathrm{V}]$ & $S[\mathrm{~V} / \mathrm{dec}]$ & $N\left[/ \mathrm{cm}^{2}\right]$ \\
\hline 20.0 & $4.9 \times 10^{-5}$ & -0.6 & 9.7 & $1.2 \times 10^{13}$ \\
11.1 & $8.8 \times 10^{-5}$ & -1.2 & 6.3 & $7.8 \times 10^{12}$ \\
5.9 & $1.6 \times 10^{-4}$ & -1.7 & 3.8 & $4.7 \times 10^{12}$ \\
0.0 & $9.9 \times 10^{-4}$ & -3.7 & 2.4 & $2.9 \times 10^{12}$ \\
\hline \hline
\end{tabular}

Table 3. Parameters obtained from NTCDA OFETs with PMMA buffer layers doped at different F4TCNQ concentrations. 